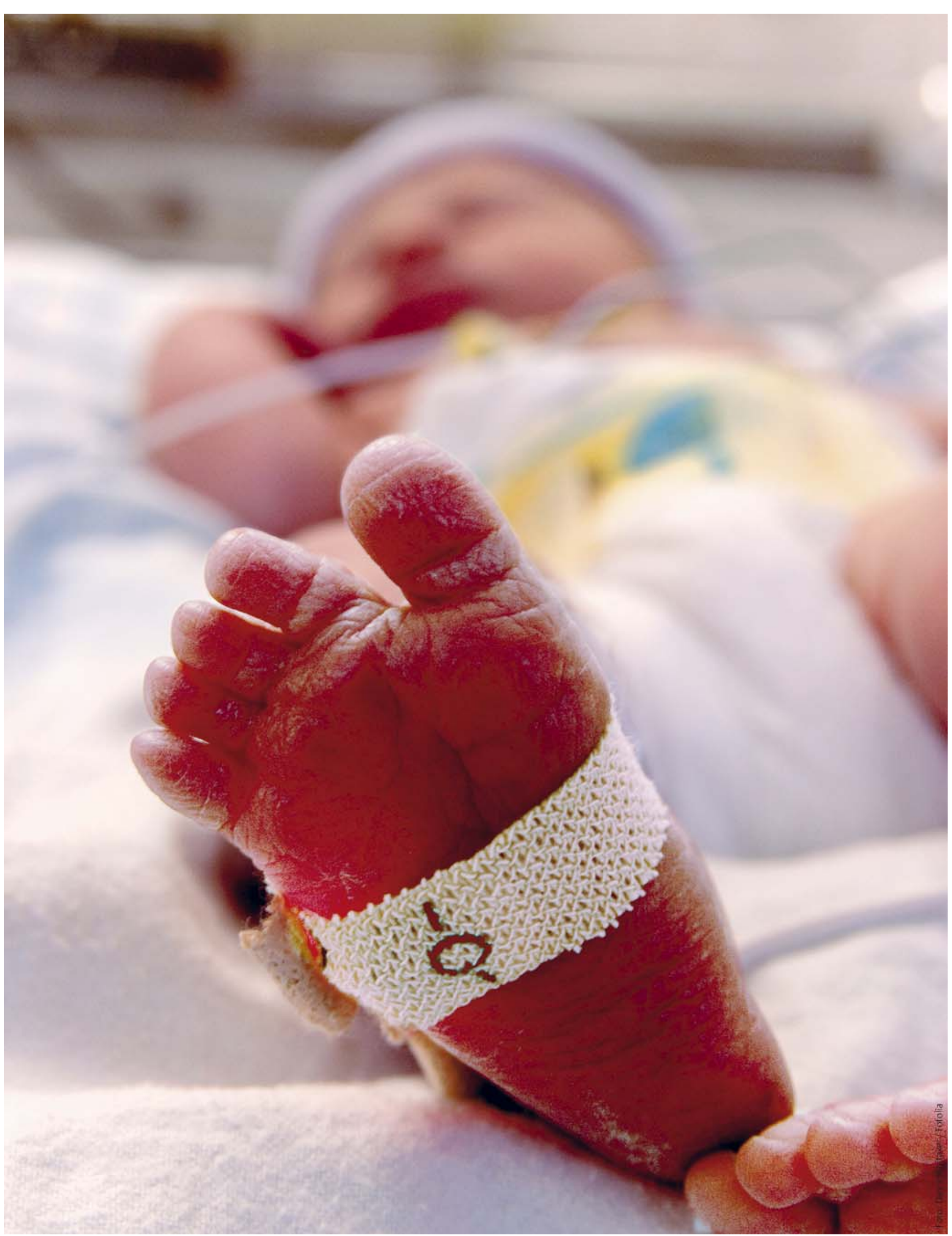




\section{Beatmung von Säuglingen und Kleinkindern: Was ist besonders?}

Gabriele Werner

\section{Kindernotfälle stellen das Personal des Rettungsdienstes häufig vor besondere He- rausforderungen. Zum einen kommen bei Kindern lebensbedrohliche Situationen wesentlich seltener vor als bei Erwachsenen und die Routine der Ersthelfer kann feh- len, zum anderen können die anatomischen und physiologischen Besonderheiten von Säuglingen und Kleinkindern zu Problemen führen [7]. Deshalb sollten Ersthelfer diese Besonderheiten kennen.}

\section{FALLBEISPIEL}

\section{Beispiel: Atemnot beim Säugling - Die Geschichte von Paul}

Der Rettungsdienst wird zu einem fünf Monate alten Jungen mit Luftnot gerufen. Er findet einen Säugling vor, der stark hustet, eine Atemfrequenz von 60/min hat und zyanotisch ist. Die Sättigung (SpO2) liegt bei Werten zwischen 82 und $85 \%$. Der Notarzt untersucht den Säugling. Dieser hat interkostale Einziehungen und ein exspiratorisches Geräusch (Giemen). Die Mutter erzählt, Paul sei fünf Monate alt, wiege sechs Kilogramm und habe seit zwei Tagen Schnupfen und trockenen Husten. Innerhalb der letzten Stunden habe sich die klinische Situation schnell verschlechtert: Paul habe Fieber bis $39^{\circ} \mathrm{C}$ entwickelt, huste viel Trachealsekret ab und die Atmung habe sich beschleunigt. Paul sei zwar in der 35. Schwangerschaftswoche zu früh zur Welt gekommen, nach einem kurzen Intensivaufenthalt zur Überwachung konnte er aber nach Hause entlassen werden und habe sich seither gut entwickelt.

\section{Atemwege}

Kinder sind keine kleinen Erwachsenen, Neugeborene erst recht nicht. Auch die Atemwege des Kindes entsprechen nicht denen eines Erwachsenen und weisen einige Besonderheiten auf [8]: Die oberen Luftwege sind nicht nur viel enger und weicher als bei Erwachsenen - auch ist die Lage der Organe zueinander anders als bei Erwachsenen [8].

Zusätzlich ändert sich die Anatomie der Atemwege im Lauf der Entwicklung eines Kindes. So sind Säuglinge unter sechs Monaten obligate Nasenatmer, die Nasenlöcher können bei einem Infekt der oberen Atemwege leicht verlegt werden. Kinder zwischen drei und acht
Jahren zeigen häufig geschwollene Rachenmandeln, die zu Atemwegsproblemen führen können. Kleinkinder haben einen vergleichsweise großen Kopf und einen kurzen Hals. Aufgrund des großen Hinterkopfs sind Intubationskissen bei Säuglingen unnötig. Eine Unterpolsterung der Schultern führt hingegen zu der für die Beatmung und Intubation günstigeren Schnüffelposition ( Abb. 1, 2).

\section{PRAXISTIPP}

Zur Unterpolsterung der Schultern kann eine Windel oder zusammengefaltete Decke genutzt werden.

Weitere Besonderheiten sind:

- Der Mundboden des Kindes ist weich und verformbar, daher kann bei der Maskenbeatmung der Atemweg leicht zugedrückt werden. Anders als beim Erwachsenen dürfen beim C-Griff die Finger nicht in den Mundboden gedrückt werden; dafür eignen sich die knöchernen Strukturen besser.

- Die relativ große Zunge kann zu Atemwegsverlegungen und zu einer erschwerten Laryngoskopie führen.

- Die Epiglottis ist bei Kleinkindern U-förmig.

- Der Kehlkopf steht bei Kindern höher (2. bis 3. Halswirbel) als beim Erwachsenen (5. bis 6. Halswirbel) [5]. Die engste Stelle des kindlichen Kehlkopfs befindet sich auf der Höhe des Ringknorpels (Cricoid), während beim Erwachsenen der Larynx die engste Stelle ist.

- Die Trachea des Kindes ist kurz und weich, beim Neugeborenen misst sie vom Kehlkopf bis zur Carina vier Zentimeter. Die einseitige Intubation ist durch die Schallweiterleitung des Atemgeräusches beim kleinen Thorax des Neugeborenen auskultatorisch nicht sicher zu erfassen. Daher muss sorgfältig darauf geachtet werden, wie tief der Tubus eingeführt wird. Eine sorgfältige Tubusfixierung ist unverzichtbar, um akzidentelle Extubationen oder endobronchiale Intubationen zu verhindern. 


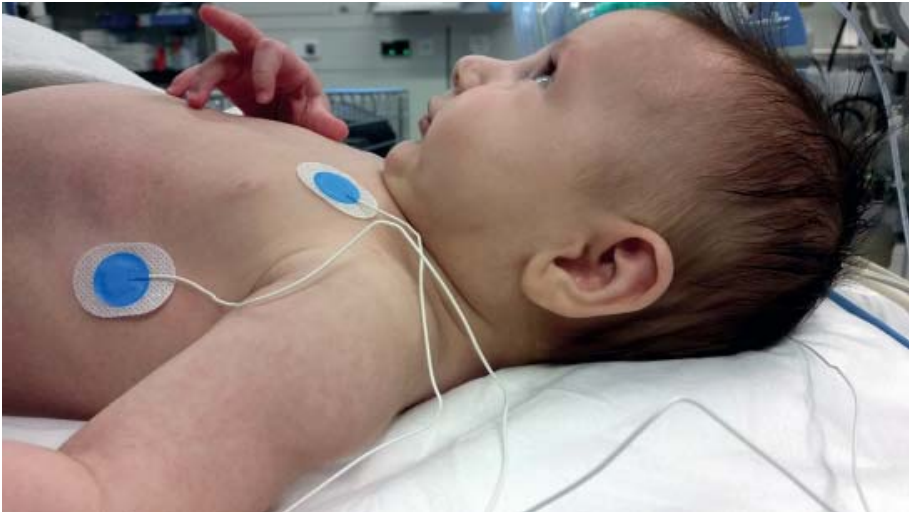

- Abb. 1 Großer Hinterkopf bei einem vier Monate alten Säugling.

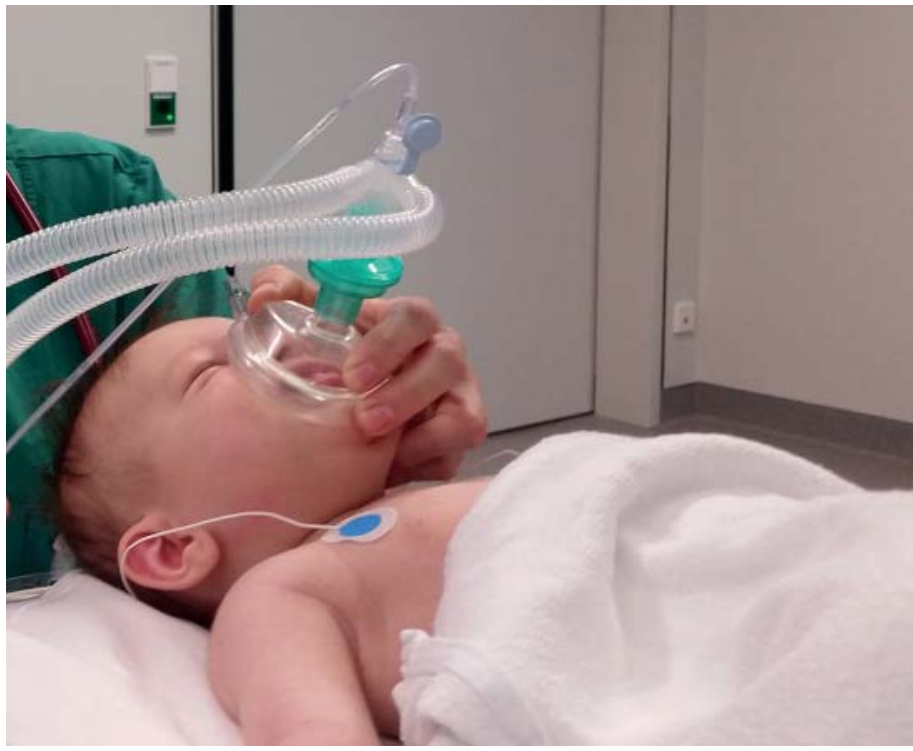

Abb. 2 Maskenbeatmung bei einem vier Monate alten Säugling in Schnüffelposition.

- Tab. 1 Atemfrequenz in Ruhe nach Lebensalter [2].

\begin{tabular}{|l|l|}
\hline Alter (in Jahren) & Atemfrequenz (Atemzüge/min) \\
\hline$<1$ & $30-40$ \\
\hline $1-2$ & $25-35$ \\
\hline $2-5$ & $25-30$ \\
\hline $5-12$ & $20-25$ \\
\hline$>12$ & $15-20$ \\
\hline
\end{tabular}

- Der Carinawinkel ist bei Kindern symmetrisch, es besteht also die Gefahr, dass sich sowohl der rechte als auch der linke Hauptbronchus durch aspirierte Fremdkörper verlegen kann.
Cave

Die einseitige Intubation ist beim Neugeborenen mit dem Stethoskop nicht immer auf Anhieb zu erfassen.

\section{FALLBEISPIEL}

\section{Pauls Geschichte geht weiter}

Der Rettungsassistent hält Paul eine Sauerstoffmaske mit Reservoir und $10 \mathrm{l} \mathrm{O2/min} \mathrm{vor,} \mathrm{über} \mathrm{die} \mathrm{er}$ Salbutamol inhaliert. Die Sättigung (SpO2) steigt aber nicht über $90 \%$. Paul bleibt weiter zyanotisch und wird zunehmend somnolent. Der Notarzt stellt die Indikation zur Intubation.

\section{Lungenfunktion}

Neugeborene und Säuglinge atmen fast ausschließlich mit dem Zwerchfell. Durch einen erhöhten intraabdominellen Druck kann es sehr schnell zur Ateminsuffizienz kommen. Daher ist bei der Maskenbeatmung unbedingt darauf zu achten, den Magen nicht aufzublähen.

\section{Cave}

Ein aufgeblähter Magen kann eine Maskenbeatmung beim Kind unmöglich machen. Der Magen muss dann sofort mit einem Absaugkatheter entlastet werden.

Der Thorax des Neugeborenen ist wesentlich elastischer und instabiler als der des Erwachsenen und kann weder einem Lungenkollaps noch einer massiven Überblähung mit Gefahr eines Barotraumas etwas entgegensetzen. Vermehrte Atemanstrengungen bei respiratorischer Insuffizienz führen zu Einziehungen.

\section{Sauerstoffverbrauch}

Der Grundumsatz von Neugeborenen und Säuglingen ist im Vergleich zum Erwachsenen deutlich erhöht. Grund dafür ist die rasche Gewichtszunahme im ersten Lebensjahr, die sich in einem erhöhten Sauerstoffbedarf zeigt $(6-7 \mathrm{ml} / \mathrm{kg} / \mathrm{min}$ im Vergleich zum Erwachsenen $3-4 \mathrm{ml} / \mathrm{kg} / \mathrm{min}$ ) und in einer zweifach erhöhten Kohlendioxidproduktion. Bei Unterkühlung kann der Sauerstoffbedarf des Neugeborenen sogar bis auf $20 \mathrm{ml} / \mathrm{kg} / \mathrm{min}$ ansteigen - das ist eine Sauerstoffmenge, die ein Neugeborenes nicht unter Spontanatmung bereitstellen kann. Da eine Unterkühlung zur Ateminsuffizienz führt, muss sie in jedem Fall verhindert werden. Selbst unter physiologischen Bedingungen ist die alveoläre Ventilation bei Neugeborenen und Säuglingen mit $100-150 \mathrm{ml} / \mathrm{kg} / \mathrm{min}$ im Vergleich zu $60 \mathrm{ml} / \mathrm{kg} / \mathrm{min}$ beim Erwachsenen deutlich höher; die Atemfrequenz des Neugeborenen beträgt $30-40 / \mathrm{min}$, die des Säuglings 30/min ( Tab. 1). Die funktionelle Residualkapazität, also das Lungenvolumen, das nach dem Ausatmen in der Lunge verbleibt, ist mit 
$30 \mathrm{ml} / \mathrm{kg}$ klein. Dies erklärt die kurze Apnoetoleranz mit geringen Sauerstoffreserven. Aus diesem Grund werden Kinder immer mit der Maske zwischenbeatmet, bis das Relaxans zur Intubation wirkt. Die modifizierte Rapid Sequence Induction (RSI) trägt der Tatsache Rechnung, dass Kinder eher hypoxie- als aspirationsgefährdet sind. Angesichts der kleinen Lungen, des erhöhten Sauerstoffverbrauchs, der Temperaturinstabilität und der stark eingeschränkten Kompensationsmöglichkeiten wird verständlich, dass Neugeborene wesentlich gefährdeter sind als Erwachsene, eine Hypoxie zu erleiden. Auf eine Hypoxie reagieren Kinder mit einem Herzfrequenzabfall.

\section{Cave}

Eine Bradykardie bei Kindern deutet meist auf eine Hypoxie hin. Die Ateminsuffizienz muss therapiert werden.

\section{PRAXIS/MABNAHMEN}

Bei Kindern wird eine modifizierte RSI (Rapid Sequence Induction) durchgeführt. Bis die Wirkung des Relaxans einsetzt, wird das Kind mit der Maske zwischenbeatmet, da es eher hypoxie- als aspirationsgefährdet ist. Schon nach kurzer Apnoe kommt es bei Kindern zu einem Sättigungsabfall.

\section{DEFINITION/SYNONYM}

Besonderheiten der Lunge des Neugeborenen [5]

- großer Sauerstoffverbrauch

- große alveoläre Ventilation

- kleine funktionelle Residualkapazität

- enge Luftwege

- instabiler Thorax

- Atemmuskulatur noch in der Entwicklung

\section{Fetaler Kreislauf}

Mit dem ersten Atemzug, wenn sich die Lungen entfalten, kommt es zur Durchblutung der Lungen: In den ersten 10 bis 15 Stunden verschließt sich der Ductus Botalli funktionell und nach vier bis sechs Wochen anatomisch. Diese Kreislaufumstellung ist in den ersten Lebenstagen noch nicht fixiert, und es kann durch Hypoxie und Azidose zur Wiedereröffnung des Ductus Botalli mit katastrophalen Folgen kommen (persistierender fetaler Kreislauf) - dies muss auf jeden Fall vermieden werden. Durch die Erhöhung des Drucks im linken Vorhof kommt es zum funktionellen Verschluss des Foramen ovale, das nach drei bis zwölf Monaten anatomisch verschlossen ist; allerdings ist bei $20 \%$ der Erwachsenen das Foramen ovale noch durchgängig [5].
- Tab. 2 Blutdruck im Kindesalter [1].

\begin{tabular}{|l|l|l|}
\hline Alter & $\begin{array}{l}\text { systolischer } \\
\text { Blutdruck }(\mathbf{m m H g})\end{array}$ & $\begin{array}{l}\text { diastolischer } \\
\text { Blutdruck } \mathbf{( m m H g})\end{array}$ \\
\hline Frühgeborene & $50 \pm 3$ & $30 \pm 3$ \\
\hline Neugeborene & $75 \pm 8$ & $50 \pm 8$ \\
\hline 6 Monate & $90 \pm 29$ & $60 \pm 10$ \\
\hline 1 Jahr & $95 \pm 30$ & $65 \pm 25$ \\
\hline 6 Jahre & $100 \pm 15$ & $55 \pm 8$ \\
\hline 12 Jahre & $110 \pm 16$ & $60 \pm 9$ \\
\hline
\end{tabular}

D Tab. 3 Herzfrequenz im Kindesalter [1].

\begin{tabular}{|l|l|l|l|}
\hline Alter & $\begin{array}{l}\text { unterer } \\
\text { Grenzwert } \\
(\mathbf{1} / \mathbf{m i n})\end{array}$ & $\begin{array}{l}\text { Mittelwert } \\
\mathbf{( 1 / m i n )}\end{array}$ & $\begin{array}{l}\text { oberer } \\
\text { Grenzwert } \\
\mathbf{( 1 / m i n )}\end{array}$ \\
\hline Neugeborene & 90 & 120 & 170 \\
\hline 1 - 12 Monate & 80 & 120 & 160 \\
\hline 2 Jahre & 80 & 110 & 130 \\
\hline 4 Jahre & 80 & 100 & 120 \\
\hline 6 Jahre & 75 & 100 & 115 \\
\hline 8 Jahre & 70 & 90 & 110 \\
\hline 10 Jahre & 70 & 90 & 110 \\
\hline
\end{tabular}

PRAXIS/MAßNAHMEN

Zur Entfaltung der Lungen werden bei der Reanimation eines Neugeborenen die fünf initialen Beatmungen mit einem konstanten Beatmungsdruck von 15 - 30 mbar durchgeführt, der über 2 - 3 Sekunden pro Beatmung anhält.

\section{Physiologische Normwerte}

Der physiologische Blutdruck und die Herzfrequenz sind abhängig vom Alter des Kindes ( $\vee$ Tab. 2, 3). Normwerte müssen bei der Versorgung von Kindern bekannt sein, um eventuelle Abweichungen auch therapieren zu können. Dabei helfen Tabellen wie das Pädiatrische Notfalllineal.

\section{Technische Umsetzung}

Ersthelfer sollten bei der Versorgung von Kindernotfällen ihre Aufmerksamkeit besonders richten auf 


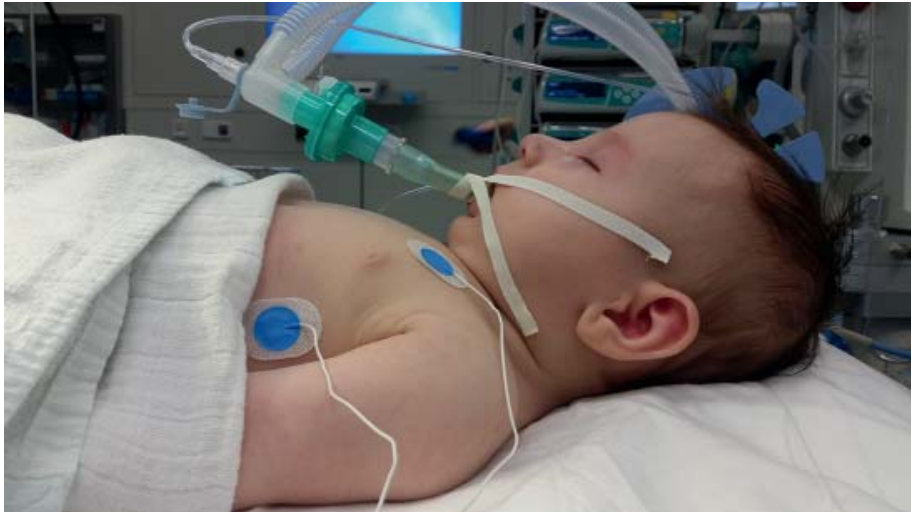

- Abb. 3 Vier Monate alter Säugling, beatmet mit einer Larynxmaske

- Tab. 4 Tubusgrößen [5].

\begin{tabular}{|l|l|l|l|}
\hline Alter & $\begin{array}{l}\text { Tubusgröße } \\
\text { (ID in mm) } \\
\text { ohne Cuff }\end{array}$ & $\begin{array}{l}\text { Tubusgröße } \\
\text { (ID in mm) } \\
\text { mit Cuff }\end{array}$ & $\begin{array}{l}\text { Einführtiefe } \\
\text { ab Zahn- } \\
\text { leiste }\end{array}$ \\
\hline $\begin{array}{l}\text { Neugebore- } \\
\text { ne 3 kg }\end{array}$ & $3,0-3,5$ & & $9 \mathrm{~cm}$ \\
\hline $\begin{array}{l}\text { 3 } \mathrm{kg}-4 \text { Mo- } \\
\text { nate }\end{array}$ & 3,5 & 3,0 & $10 \mathrm{~cm}$ \\
\hline $\begin{array}{l}\text { 4-12 Mo- } \\
\text { nate }\end{array}$ & 4,0 & 3,0 & $11 \mathrm{~cm}$ \\
\hline 1-2 Jahre & $4,5-5,0$ & 3,5 & $12-13 \mathrm{~cm}$ \\
\hline 2-4 Jahre & $5,0-5,5$ & 4,0 & $13-14 \mathrm{~cm}$ \\
\hline 4-6 Jahre & $5,5-6,0$ & 4,5 & $14-15 \mathrm{~cm}$ \\
\hline 6-8 Jahre & $6,0-6,5$ & 5,0 & $15-16 \mathrm{~cm}$ \\
\hline 8-10 Jahre & & 5,5 & $16-17 \mathrm{~cm}$ \\
\hline 10 - 12 Jahre & & 6,0 & $18-19 \mathrm{~cm}$ \\
\hline
\end{tabular}

- die Sicherung der Atemwege,

- die Anlage von Zugängen und

- die Dosierung der Medikamente.

Grundsätzlich sollten alle Helfer Verfahren, Instrumente und Medikamente vorziehen, die ihnen vertraut sind. Bleibt mit der gewählten Methode der Erfolg aus, müssen alle Beteiligten frühzeitig an eine Alternative denken. Bei einer Ateminsuffizienz hat nicht die Intubation den höchsten Stellenwert, vorrangig wichtig ist die Beatmung des Kindes, um sicherzustellen, dass die lebenswichtigen Organe ausreichend mit Sauerstoff versorgt werden (Oxygenierung). Ob dies mit der Maskenbeatmung gelingt, oder ob supraglottische Atemwegshilfen wie Larynxmaske ( $\triangleright$ Abb. 3 ) oder -tubus zum Zuge kommen oder oral oder nasal intubiert wird, hängt von unterschiedlichen Faktoren ab: der speziellen Situation, der Anatomie des Kindes, aber auch von der Erfahrung und dem Können des Helfers. Um den Stress im Notfall zu minimieren, empfiehlt es sich, ein Nachschlagewerk, ein Kindermaßband oder Notfallineal auf dem RTW oder NEF zu installieren. Es gibt eine Vielzahl solcher Systeme und Maßbänder - und auch bei der Wahl des passenden Endotrachealtubus können diese genutzt werden. Für die klinische Praxis kann man sich für den Außendurchmesser des Tubus ganz grob an der Dicke des kleinen Fingers orientieren - dieses Verfahren hält jedoch wissenschaftlichen Überprüfungen nicht stand. Die Formel „Alter/4 + 4“ ist für Kinder mit einem Lebensalter über 2 Jahren validiert und gilt für den Innendurchmesser von nicht geblockten Tuben für Kinder ab einem Jahr. Wird ein Tubus mit Cuff eingesetzt, soll eine halbe Nummer kleiner gewählt werden ( $\$$ Tab.4). Wichtig ist, jede Kraftausübung zu unterlassen - vor allem unterhalb der Stimmritze - und bei Bedarf auf eine kleinere Tubusgröße zu wechseln [7].

Gelingt es bei vitaler Indikation nicht, einen venösen Zugang zu legen, soll eine intraossäre Nadel gelegt werden. Verschiedene Nadelsysteme sind auf dem Markt; die semiautomatischen Systeme sind wesentlich schneller als die manuell einzubohrenden Nadeln. Die erste Wahl als Zugangsweg stellt die proximale Tibia dar, 1 $2 \mathrm{~cm}$ medial und distal der Tuberositas tibiae. Weitere Punktionsorte sind der distale Femur, die distale Tibia, die Spina iliaca anterior superior und der proximale Humerus. Wichtig: Bein Legen der intraossären Nadel sollten die Epiphysenfugen nicht verletzt werden.

\section{FALLBEISPIEL}

\section{Paul wird transportfähig}

Während der Rettungsassistent die Intubation vorbereitet, legt der Notarzt eine 24-G- Venenverweilkanüle in Pauls rechten Handrücken. Das Kind ist mittlerweile somnolent und wird unterstützend mit einem Ambubeutel mit Reservoir und 10I Sauerstoff beatmet. Man entscheidet sich für die Tubusgröße 4,0 mm I.D. ohne Cuff und legt die Größe 3,5 mm I. D. bereit. Der Rettungsassistent appliziert $0,01 \mathrm{mg}$ Fentanyl ( $1-2 \mu \mathrm{g} / \mathrm{kg} \mathrm{KG),} 25 \mathrm{mg}$ Propofol $(4 \mathrm{mg} / \mathrm{kg}$ KG). Die Maskenbeatmung ist zunächst erschwert. Der Rettungsassistent unterpolstert mit einer Windel die Schultern. In dieser Schnüffelposition und nach Einlegen eines Guedel-Tubus ist die Maskenbeatmung möglich. Relaxiert wird Paul mit $0,06 \mathrm{mg}$ Vecuronium (0,1 mg/kg KG). Die Sauerstoffsättigung steigt auf $94 \%$. Die Intubation gelingt mit einem Tubus 4,0 mm I.D. problemlos, der Rettungsassistent fixiert den Tubus sorgfältig bei $11 \mathrm{~cm}$ Einführtiefe. Paul wird intubiert und beatmet in die nächstgelegene Kinderklinik und auf die Intensivstation gebracht. Dort wird die Diagnose einer RSV-Infektion (Respiratory Syncytial Virus) gestellt - eine Infektion der Atemwege, die vor allem bei Frühgeborenen zu schweren Verläufen führen kann. Paul kann nach 
wenigen Tagen intensivmedizinischer Therapie extubiert werden.

\section{KERNAUSSAGEN}

1. Kindernotfälle können im Rettungsdienst eine besondere Herausforderung darstellen. Lebensbedrohliche Situationen sind selten, es kann die Routine fehlen, und die anatomischen und physiologischen Besonderheiten von Kindern können zu Problemen führen.

2. Besonderheiten der Atemwege des Kindes sind u. a. eine große Zunge, ein weicher Mundboden, ein hochstehender Kehlkopf und eine U-förmige Epiglottis. Wegen des großen Hinterkopfes ist die Schnüffelposition die optimale Lagerung zur Maskenbeatmung und Intubation.

3. Säuglinge atmen fast ausschließlich mit dem Zwerchfell. Ein aufgeblähter Magen kann eine Maskenbeatmung beim Kind unmöglich machen. Der Magen muss dann mit einem Absaugkatheter entlastet werden.

4. Säuglinge haben im Vergleich zum Erwachsenen einen deutlich erhöhten Sauerstoffverbrauch, der bei Unterkühlung noch weiter ansteigt und meist zur Ateminsuffizienz führt.

5. Säuglinge haben eine kurze Apnoetoleranz mit nur geringen Sauerstoffreserven. Daher werden Kinder vor der Intubation bis zu dem Zeitpunkt, zu dem die Wirkung des Relaxans einsetzt, immer mit der Maske beatmet.

6. In den ersten Lebenstagen kann es durch Hypoxie und Azidose zur Wiedereröffnung des Ductus Botalli kommen.

7. Physiologische Normwerte sollen bekannt sein, um Abweichungen therapieren zu können. Hilfreich dafür können Maßbänder oder Taschenkarten sein.

8. Wenn bei vitaler Indikation das Legen eines venösen Zugangs nicht gelingt, soll eine intraossäre Nadel gelegt werden.

9. Bei einer Ateminsuffizienz ist die Oxygenierung lebenswichtiger Organe wichtigstes Ziel. Ob dies durch Maskenbeatmung, Intubation oder supraglottische Atemwegshilfen ermöglicht wird, ist abhängig von der Situation, der Anatomie des Patienten und dem Können des Helfers.

10. Bei der richtigen Dosierung der Medikamente sind Maßbänder oder Taschenkarten sinnvoll. Sie minimieren im Notfall nicht nur den Stress, sondern senken auch die Gefahr von Fehldosierungen.

\section{Interessenkonflikt}

Die Autoren geben an, dass kein Interessenkonflikt besteht.

\section{Über die Autoren}

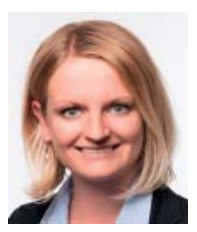

\section{Dr. Gabriele Werner,}

2002-2008 Studium der Humanmedizin an der Universität des Saarlandes. 2009-2015 Klinik für Anästhesiologie und operative Intensivmedizin, Universität Witten/Herdecke, Köln- Merheim. 2012 Notärztin der Stadt Köln. 2012 Zusatzbezeichnung Notfallmedizin. 2014 Facharztprüfung. 2015 Zusatzbezeichnung Spezielle Intensivmedizin. Seit 2012 freiberufliche Notärztin und seit 2016 tätig für die Kliniken der Stadt Stuttgart, Olgahospital.

Korrespondenzadresse

Dr. med. Gabriele Werner

Oberärztin für Anästhesiologie, Klinikum Stadt Stuttgart, Olgahospital/Frauenklinik

Kriegsbergstr. 60

70174 Stuttgart

ga.werner@klinikum-stuttgart.de

\section{Literatur}

[1] Becke K, Eberius C, Siebert C et al. Memorix Kinderanästhesie. Stuttgart: Thieme. 2011

[2] Brokmann J, Rossaint R. Repetitorium Notfallmedizin. Berlin, Heidelberg: Springer. 2010; 2. Aufl

[3] Ellinger K, Grenzwürker H. Kursbuch Notfallmedizin. Köln: Deutscher Ärzte-Verlag. 2011; 2. Aufl

[4] Höhne C. Management des kindlichen Atemwegs. Anästh Intensivmed 2008; 49: 65-74

[5] Jöhr M. Kinderanästhesie. München: Urban \& Fischer. 2009; 7. Aufl

[6] Jöhr M. Kleine Kinder - große Probleme. Anästh Intensivmed 2005; 46: $358-372$

[7] Kaufmann J, Laschat M, Wappler F. Die präklinische Versorgung von Notfällen im Kindesalter. Anästh Intensivmed 2012; 53: $254-267$

[8] Kretz F], Becke K. Anästhesie und Intensivmedizin bei Kindern. Stuttgart: Thieme. 2007; 2. Aufl

\section{Bibliografie}

DOI https://doi.org/10.1055/s-0043-101494

retten 2017; 6: 211-217

(C) Georg Thieme Verlag KG Stuttgart · New York ISSN 2193-2387 
Punkte sammeln auf CEE.thiemede

Diese Fortbildungseinheit ist 12 Monate online für die Teilnahme verfügbar.

Sollten Sie Fragen zur Online-Teilnahme haben, finden Sie unter cme.thieme.de/hilfe

eine ausführliche Anleitung. Wir wünschen viel Erfolg beim Beantworten

der Fragen!

Unter https://eref.thieme.de/ZZX8L1Z oder über den QR-Code kommen Sie direkt zum Artikel zur Eingabe der Antworten.

\section{Frage 1}

Welche Aussage zum kindlichen Atemweg ist falsch?

A Die Epiglottis ist U-förmig.

B Die Trachea ist kurz und weich.

C Der Mundboden ist weich und verformbar.

D Die engste Stelle beim kindlichen Atemweg ist der Larynx.

E Die große Zunge kann zu Atemwegsverlegungen führen.

\section{Frage 2}

Welche Aussage zur Beatmung Neugeborener ist falsch?

A Der Thorax des Neugeborenen ist instabiler als der des Erwachsenen.

B Aufgrund der Elastizität des Thorax ist ein Barotrauma während der Beatmung eher selten.

C Bei einer Ateminsuffizienz ist die Oxygenierung das wichtigste Ziel, die angewendeten Verfahren hängen u. a. von der Erfahrung des Anwenders ab.

D Bei einer schwierigen Intubation kann auf supraglottische Atemwegshilfen zurückgegriffen werden.

E Neugeborene haben eingeschränkte Kompensationsmöglichkeiten.

\section{Frage 3}

Welche dieser fünf Aussagen trifft bei Säuglingen nicht zu?

A Der Sauerstoffverbrauch ist höher als der des Erwachsenen.

B Säuglinge sind wesentlich gefährdeter als Erwachsene, eine Hypoxie zu erleiden.

C Säuglinge haben nur eingeschränkte Kompensationsmechanismen.

D Der Grundumsatz des Säuglings ist im Vergleich zum Erwachsenen deutlich erhöht.

E Säuglinge tolerieren eine Apnoezeit von vier Minuten problemlos.

\section{Frage 4}

Welche Aussage zum Blutdruck eines Säuglings stimmt nicht?

A Der physiologische Blutdruck ist abhängig vom Alter des Kindes.

B Die Atemfrequenz bei Säuglingen beträgt 30-40 Atemzüge/ Minute.

C Der Blutdruck eines sechs Monate alten Säuglings beträgt durchschnittlich $90 / 60 \mathrm{mmHg}$.

D Eine Herzfrequenz von 60 Schlägen pro Minute bei einem Neugeborenen ist physiologisch.

E Normwerte müssen bei der Versorgung von Kindern bekannt sein, um Abweichungen therapieren zu können.

\section{Frage 5}

Welche Aussage zur Maskenbeatmung trifft nicht zu?

A Zur Maskenbeatmung muss der Kopf des Säuglings in die sogenannte Schnüffelposition gebracht werden.

B Eine Unterpolsterung der Schultern kann bei der Maskenbeatmung helfen.

C Bei der Maskenbeatmung kann es zu einem Aufblähen des Magens kommen.

D Wenn die Maskenbeatmung infolge eines aufgeblähten Magens unmöglich ist, soll mit höheren Beatmungsdrücken eine ausreichende Ventilation sichergestellt werden.

E Bei Problemen mit der Maskenbeatmung kann ein GuedelTubus helfen.

\section{Frage 6}

Welche Aussage zur Intubation ist richtig?

A Die Wahl des passenden Tubus hängt vom Gewicht des Kindes ab.

B Bei Intubationsproblemen sollte der Hinterkopf des Säuglings unterpolstert werden, um eine bessere Sicht auf den Larynx zu erhalten.

C Bei einem reifen Neugeborenen sollte der Tubus (oral) bei circa $9 \mathrm{~cm}$ fixiert werden.

D Die korrekte Tubuslage kann beim Säugling problemlos durch Auskultation bestätigt werden.

E Die korrekte Tubusgröße kann mit der Formel „Alter/2+4“ errechnet werden.

\section{- Weitere Fragen auf der folgenden Seite...}




\section{CME-Fragen bei CMIEthieme.de}

Fortsetzung $\ldots$

Frage 7

Die Atmung von Säuglingen und Kindern unterscheidet sich von der der Erwachsenen. Welche der folgenden Aussagen ist falsch?

A Das Atemzugvolumen ist mit $6 \mathrm{ml} / \mathrm{kg}$ in allen Altersklassen gleich.

B Neugeborene und Säuglinge atmen fast ausschließlich mit dem Zwerchfell.

C Kinder reagieren auf eine Hypoxie mit einer Steigerung der Herzfrequenz, damit mehr Sauerstoff zu den Organen transportiert werden kann.

D Vermehrte Atemanstrengung bei respiratorischer Insuffizienz führt zu intercostalen Einziehungen.

E Bei Unterkühlung kann der Sauerstoffbedarf des Neugeborenen bis auf $20 \mathrm{ml} / \mathrm{kg} / \mathrm{min}$ ansteigen.

\section{Frage 8}

Welche Aussage ist falsch?

A Wenn bei vitaler Indikation das Legen eines venösen Zugangs nicht möglich ist, soll eine intraossäre Nadel gelegt werden.

B Kinder sollen bei der Intubation immer mit der Maske zwischenbeatmet werden.

C Wird ein Tubus mit Cuff gewählt, soll dieser eine halbe Nummer kleiner sein als der Tubus ohne Cuff.

D Für einen Neugeborenen soll ein Tubus ohne Cuff der Größe 4,0 ID mm gewählt werden.

E Für einen 6 Monate alten Säugling ist eine Tubusgröße von 3,0 ID mm mit Cuff die richtige Wahl.

\section{Frage 9}

Die Lunge eines Neugeborenen weist Besonderheiten auf. Eine der folgenden Aussagen trifft nicht zu. Welche?

Die Besonderheiten der Lunge des Neugeborenen sind:
A Enge Luftwege
B Instabiler Thorax
C Atemmuskulatur noch in Entwicklung
D Kleine aalveoläre Ventilation
E Großer Sauerstoffverbrauch

\section{Frage 10}

\section{Welche Aussage zur RSV-Infektion ist richtig?}

A Eine RSV-Infektion kommt erst ab dem Kindergartenalter vor.

$B$ Eine RSV-Infektion führt bei ehemaligen Frühgeborenen eher selten zu schweren Verläufen.

C Symptome sind zunächst Schnupfen und trockener Husten, es kann innerhalb kurzer Zeit zu respiratorischer Insuffizienz kommen.

D Zeichen einer respiratorischen Insuffizienz sind bei Erwachsenen u. a. Einziehungen am Thorax.

E Der Notarzt hätte Paul in dieser Situation nicht intubieren müssen, Paul hätte sich spontan wieder erholt. 